\title{
Practical requirements for modelling the dynamics of hydraulic pipelines
}

\author{
K. SANADA*, C. W. RICHARDS, D. K. LONGMORE, D. N. JOHNSTON and C. R. BURROWS \\ Fluid Power Centre, School of Mechanical Engineering, University of Bath, United Kingdom \\ * visiting fellow from Department of Control Engineering, Faculty of Engineering, Tokyo Institute of Technology, \\ Japan
}

\begin{abstract}
Hydraulic pipeline dynamics play an important role in many real systems, for example in Diesel fuel injection, anti-skid braking, under-sea oil production and hydraulic control in general. When investigating the behaviour of such systems by simulation it is necessary to have suitable numerical models for the line. An excessively complicated model wastes computing time, but an inadequate one fails to predict the behaviour satisfactorily. A first step in deciding upon a suitable model is to look at the relationship between the frequency content of transient operations in the system and the natural frequencies of the lines. Rapid transients can be initiated by such things as valve operation, actuators reaching the end of their travel and cavitation and air release. A second important criterion is the amount of damping in the lines caused by fluid friction. This may be steady or frequency dependent friction in laminar or turbulent flow. On the one hand, increasing the complexity of the friction model employed makes the computations more involved. On the other hand, friction may limit the amount of higher frequency motion and consequently make the computations simpler. This paper examines the requirements for line models under different circumstances, and how these requirements can be met. Discussions are illustrated with results on practical systems.
\end{abstract}

\section{NOMENCLATURE}

$A \quad$ pipe cross sectional area

$B \quad$ fluid bulk modulus

$c \quad$ speed of sound

$D_{n} \quad$ Dissipation number

$f \quad$ steady turbulent friction factor

$L \quad$ pipe length

$p \quad$ pressure

$p_{f} \quad$ friction term

$P_{n} \quad$ Propagation number

$q \quad$ flowrate

$R \quad$ pipe internal radius

$t \quad$ time

$u \quad$ dummy variable

$W \quad$ Zielke's weighting function

$x \quad$ distance along pipe

$v \quad$ kinematic viscosity

$\rho \quad$ fluid density

$\boldsymbol{\omega}$

angular frequency

\section{INTRODUCTION}

When simulating the dynamic response of hydraulic systems, the transient behaviour of fluid in pipelines can have a significant effect. The sort of behaviour that can occur can be illustrated by a simulation of the simple hydraulic circuit shown in FIGURE 1. For this simulation an optimized finite element model which will be referred to later, and which included frequency dependent friction, was used to model the pipe.

A step change of demand current was fed to the control valve. The valve opened with a short delay due to its dynamics, as shown in FIGURE 2. The rapid opening of the valve caused a wave to be generated and to propagate along the hydraulic line. This can be seen in the early part $(t<0.16 \mathrm{~s})$ of the transient pressure at the piston end of the line. The piston then starts to lift the load. As seen from the latter part of the pressure trace shown in FIGURE $2(t>0.16 \mathrm{~s})$, when the actuator reaches the end of its travel, it stops suddenly, causing further transient pressure fluctuations. 


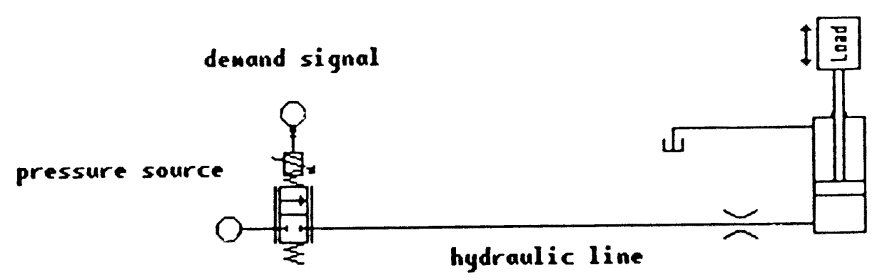

control value

FIGURE 1 A typical oil hydraulic system
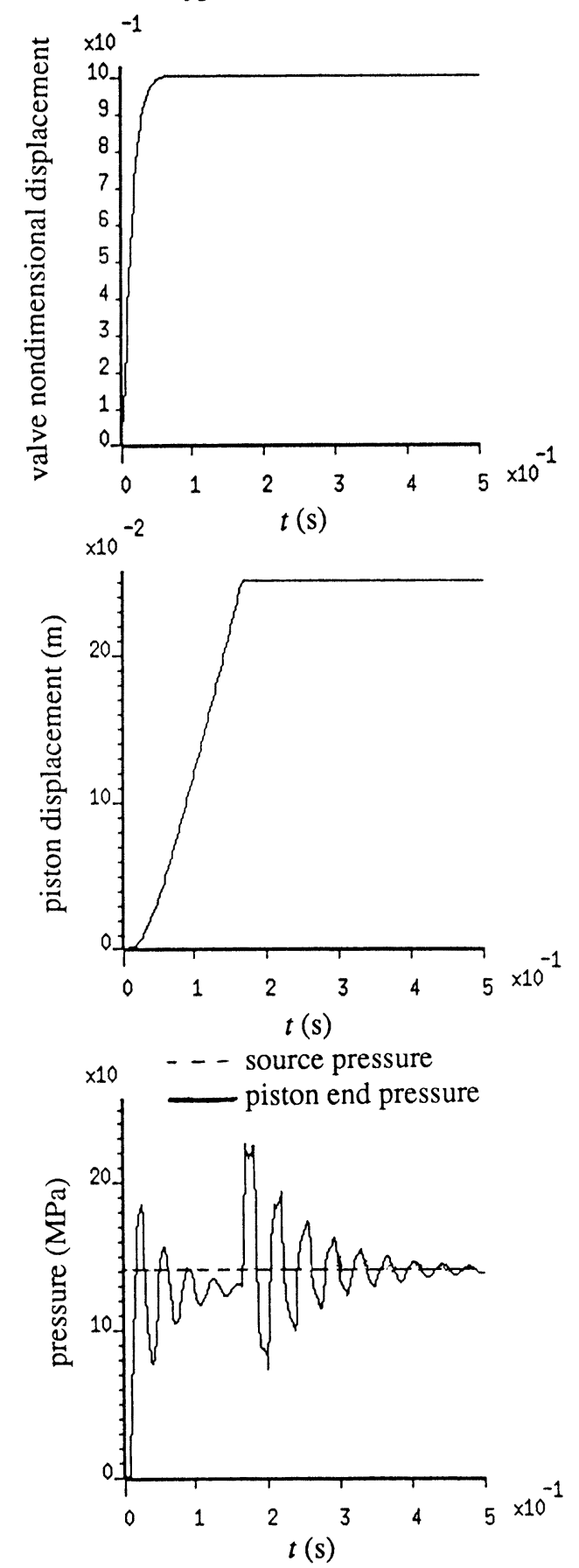

FIGURE 2 Simulated response of valve-actuator system
The piston motion is relatively smooth. The response characteristics of components can serve to limit the effects of rapid fluid transients on the components themselves. However, the transient pressure in the fluid can become very high. In FIGURE 2 , the maximum pressure is $23 \mathrm{MPa}$ which is much higher than the source pressure of $14 \mathrm{MPa}$.

actuat or Criteria for choosing suitable numerical models with which to simulate pipeline dynamics are discussed in this paper.

\section{MODELS OF HYDRAULIC PIPELINES}

\section{Basic Equations}

As a basis for the analysis of hydraulic pipelines, one dimensional flow is normally assumed. Usually the flow velocity is sufficiently low for the convection term to be neglected. Based on these assumptions, the equation of motion and continuity equation are written as follows [1] .

$$
\begin{aligned}
& \frac{\partial q}{\partial t}+\frac{A}{\rho} \frac{\partial p}{\partial x}+p_{f}(q)=0 \\
& \frac{\partial p}{\partial t}+\frac{\rho c^{2}}{A} \frac{\partial q}{\partial x}=0
\end{aligned}
$$

For laminar flow, the friction term $p(q)$ is written as follows [2] .

$$
p_{f}(q)=\frac{8 v}{R^{2}} q+\frac{4 v}{R^{2}} \int_{0}^{t} W(t-u) \frac{\partial q}{\partial t}(u) \mathrm{d} u
$$

The first term on the right hand side represents steady friction. The second term is an unsteady or frequency dependent friction term. For turbulent flow, considering steady friction only, the friction term is written as follows.

$$
p_{f}(q)-\frac{f}{4 \pi R^{3}} q|q|
$$

\section{Lumped Parameter Models}

Inertia of fluid in a pipe will be negligible if rates of change of flow are sufficiently small. Compressibility will be negligible if, in addition, other series stiffnesses are much less than that of the fluid column in the pipe, such as when the pipe is connected to much larger fluid volumes. If compressibility of fluid in a pipe is unimportant, all of the fluid will move with the same 
flow rate and therefore flow resistance can be lumped, and also inertia, if significant; only a single coordinate is necessary to describe the motion.

In circumstances where compressibility cannot be neglected, it may still be possible to lump it as a single undistributed parameter. An $\mathrm{I}+\mathrm{R}+\mathrm{C}$ network model is a combination of lumped inertia, resistance and compressibility models connected in a form analogous to an electrical network.

\section{Distributed Models}

A better approximation to distributed parameters along a pipeline can be achieved by using a series of $\mathrm{I}+\mathrm{R}+\mathrm{C}$ networks. However, there are more efficient ways of improving the numerical representation.

The method of characteristics has been widely used in many practical problems. The equation of motion and continuity equation are transformed to a set of characteristic equations along characteristic lines [1]. If the mesh is generated without any approximation, wave propagation can be modelled very accurately. Various forms of friction can be used. Unsteady friction can readily be modelled [2]. However, because the calculation is usually performed using a fixed time step, there is often difficulty in determining a compatible time step and boundary conditions [1].

Another approach is the modal approximation technique, derived from the concept of an impedance matrix used in the frequency domain [3]. A truncated number of normal modes of the pipeline are represented by use of second order lag elements with corresponding natural frequencies and damping ratios. Frequency dependent friction in laminar flow can be included. However it is important to realise that the origin of the modal approximation model is an impedance matrix which is basically a linearized model, and therefore a linear friction model is assumed. Non-linear effects cannot be modelled.

The other two standard methods of reducing the degrees of freedom of a continuously distributed system to a finite and manageable number are the finite difference method [4] and the finite element method [5]. In both of these methods, the truncated set of normal modes which can be calculated for any particular boundary conditions may show significant deviation from the true modes of the corresponding continuous pipeline. In order to minimise this problem, an optimized finite element model has been developed by the authors. From the equation of motion and the continuity equation, the model is developed using an interlacing grid system. The grid spacing is optimized to make the undamped natural frequencies of the model as close as possible to those of a continuously distributed pipeline for all the pairs of extreme boundary conditions of no flow or no pressure. This model adopts various forms of friction, including frequency dependent friction in laminar flow. The model is a set of ordinary differential equations and therefore it allows a numerical integrator with an adaptive time step scheme to be used. Different pipeline physical properties can be used in different elements, and they can be changed from step to step in response to changing conditions.

\section{CRITERIA FOR AN ADEQUATE MODEL}

The truncation of normal modes by the discretisation procedures reviewed in the preceding section, and the possibility of discretisation errors increasing progressively from the lowest to the highest mode, are effects which may manifest themselves in a time domain simulation, usually in the form of spurious apparent oscillations when pressure, flow and other parameters are plotted against time. Three factors determine whether or not this is likely to be a problem, and these are discussed in this section.

If it is necessary to model a very large number of modes, it may be more appropriate to use the method of characteristics. One would then use a fixed short integration time step, which might introduce problems. In many cases, the criteria discussed below will allow a comparatively crude spatial discretisation to be employed. Use of a variable time step integrator can then provide an efficient and fast simulation.

\section{Frequency Content of the Excitation}

In the example described in the introduction, if the valve had been opened more slowly, or if the cylinder had been effectively cushioned at the end of its travel, the high frequency content of the generation process would have been reduced. If there is negligible excitation above a certain frequency, it is unnecessary to have a model which accurately reproduces normal modes with frequencies higher than this. This provides a useful guide to the number of degrees of freedom required in numerical models.

The frequency content of an input could be plotted against frequency, but in order to relate the excitation to potential pipe models, it is convenient to plot against frequency divided by the lowest natural frequency of the fluid column in a pipeline, which occurs when one end is closed and the other open (constant pressure). This has been termed the propagation number [6] for a pipe and is defined by the equation

$$
P_{n}=\frac{2 \omega L}{\pi c}
$$


An input containing frequency components corresponding to high propagation numbers will potentially require a numerical model with a large number of elements or stations.

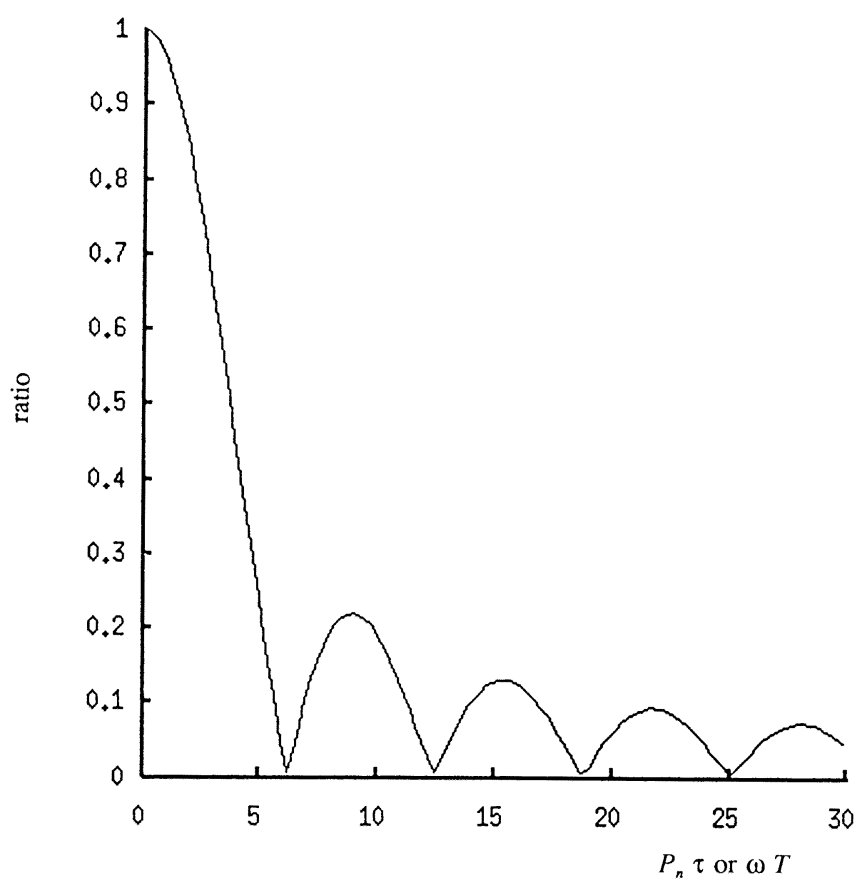

FIGURE 3 Ratio of frequency content of step with a slope to that of an instantaneous step change

The motion of a valve, or the motion of an actuator at the end of its travel, may have a variety of forms. However, FIGURE 3 is a simple illustration of how the rapidity of operation or cushioning affects the frequency content of an excitation. It is assumed here that the motion is a ramp change with a constant slope for a time $T$, followed by a constant value, and the frequency content of such a signal is compared to that of an instantaneous step by plotting the magnitude of the ratio of their Fourier transforms. The abscissa is $\omega$ $T$, which can also be written $P_{\mathrm{n}} \tau$, where $\tau$ is the ratio of the duration of the slope to the fundamental period of the pipeline. The frequency content of an instantaneous step decreases as the reciprocal of frequency, which provides a worst case. It can be seen that introducing a finite initial slope cuts down the higher frequencies by an amount dependant on the duration of the ramp. If the first zero in FIGURE 3 is taken as an acceptable limit above which frequency components can be neglected, this implies that a numerical model should be capable of accurately calculating normal modes of the pipeline up to $6 / \tau$ times the fundamental, and therefore that it should have an appropriate number of degrees of freedom. For greater precision at the expense of longer computing times, it might be desirable to have a higher limit than this.

Cavitation and air release may be quite rapid. Mechanically produced excitation, such as from valves, may have less high frequency content and may only necessitate a distributed model when lengthy pipework is involved.

For the many applications in which the frequency content of the input is low, use of a distributed model can be undesirable for two reasons. The obvious one is that it involves a lot more computation. Another reason is that it is often convenient to use instantaneous step changes in a simulation to represent variations from one level to another which are in reality much smoother. Under these circumstances, simple lumped parameter models filter out the high frequency oscillations which otherwise would be generated in the simulation but which would be absent in the physical system.

\section{Attenuation of Higher Frequencies by Fluid Friction}

Another upper limit on the frequencies which a model has to represent with acceptable accuracy is provided by fluid friction or damping. Because of the inertia of the fluid, the velocity profile across a pipe changes when the acceleration becomes large. Mathematically, this is taken into account by equation (3). It makes frictional losses greater at high frequency and this can be particularly helpful in rapidly reducing higher frequency components to negligible proportions. The modelling of frequency dependant friction can greatly increase the amount of computation, but in a finite element model this is offset by the reduced number of elements required.

The dissipation number [6] is a non-dimensional measure of fluid friction which determines how much a wave will be attenuated.

$$
D_{n}=\frac{v L}{c R^{2}}
$$

This can be seen by simplifying the typical hydraulic circuit of FIGURE 1, and considering a blocked end pipe. Transient pressures at the blocked end per unit step increase of inlet pressure are presented in FIGURE 4 with various dissipation numbers. Time is nondimensionalized by the wave travelling time, and frequency dependent friction in laminar flow is used.

\section{Filtering of Higher Frequencies by Other Components}

When considering the complexity of pipe model required in a simulation it is important to look at other parts of the system to see whether these may limit the 


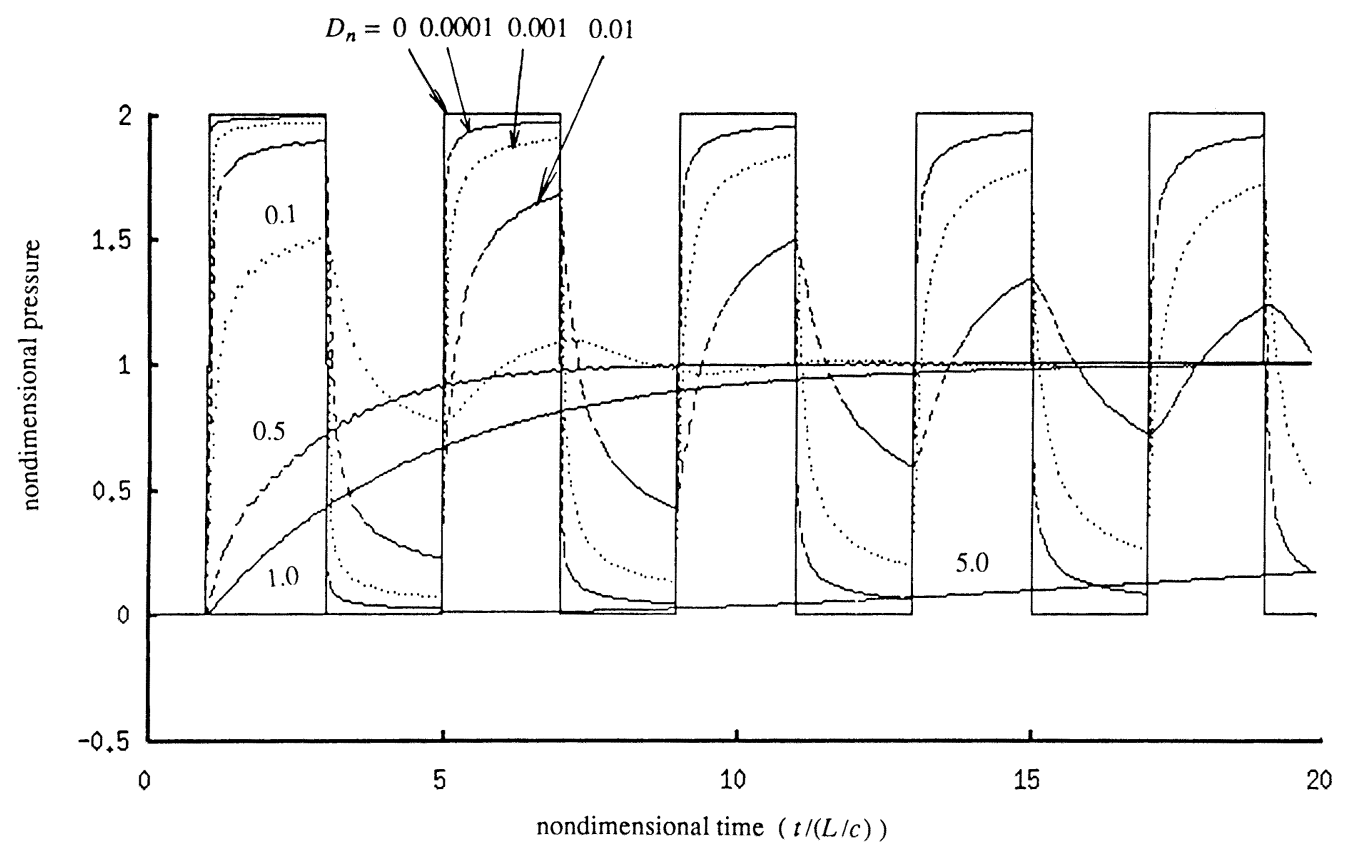

FIGURE 4 Effect of dissipation number on pipe step

response

frequency content in a pipe. For example, the sources of high frequency generation may be separated from pipes by large volumes which may act as filters.

Where the interest is in the motion of an actuator, for example, which is not likely to respond to high frequencies, it may still be important to know of any large pressure fluctuations occurring in a pipeline.

\section{ILLUSTRATIVE EXAMPLES}

\section{Long Pipelines}

The behaviour of very long pipelines is illustrated by a typical circuit used for controlling shut-off valves at an undersea oil well-head (FIGURE 5). Typically, a hose of length 0.5 to $25 \mathrm{~km}$ is pressurised to $20 \mathrm{MPa}$. The pressure maintains a valve in the open position on the sea bed by means of an actuator. A valve on the platform at the surface is used to control the system pressure. Because of the great length of the hose, when the control valve is operated at the surface, the valve on the sea bed may take several minutes or even hours to close.

Simulations were performed to determine the time taken to depressurize a hose of length $2000 \mathrm{~m}$ and bore $6.35 \mathrm{~mm}$. In this example, the system was simplified by representing the actuator as a blocked end. A step change in pressure was assumed at the surface valve. The density and kinematic viscosity of the hydraulic oil were $855 \mathrm{~kg} / \mathrm{m}^{3}$ and $47 \mathrm{cSt}$

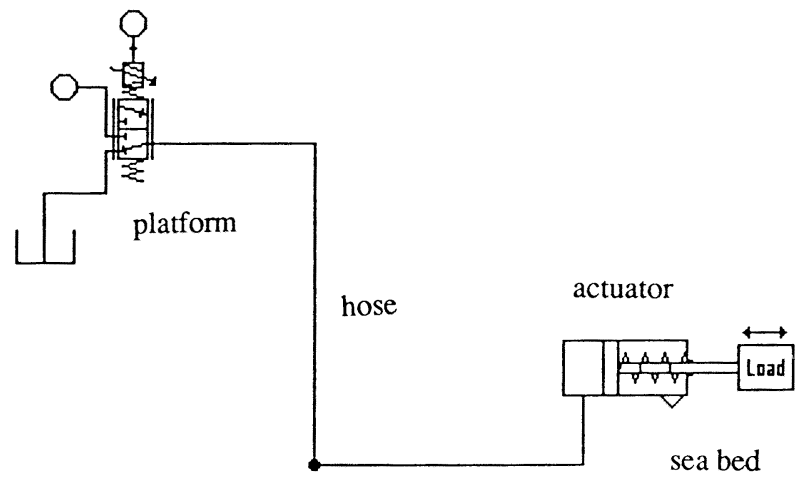

FIGURE 5 Long sub-sea hose system

respectively. Anticipating that inertia of both the oil and the hose wall will be comparatively unimportant, an effective bulk modulus is used for the fluid which includes hose expansibility. This depends on pressure and varies from $190.8 \mathrm{MPa}$ at atmospheric pressure to $712.8 \mathrm{MPa}$ at a pressure of $50 \mathrm{MPa}$. The dissipation number of the hose is large, varying from 10.16 to 19.76 , so friction is a dominant factor.

Pressures at the blocked end, i.e. at the actuator on the sea bed, are shown in FIGURE 6. Initially, a simple lumped parameter model including resistance and compressibility was tested. After opening the valve on the platform, actuator pressure reduced, taking more than $500 \mathrm{~s}$ to decay. Two kinds of finite difference models using interlacing grid systems were also tested. Initially the effective bulk modulus was assumed constant at $517 \mathrm{MPa}$. Inertia of hydraulic oil in the hose was considered in the distributed model. 
However, in model 3 , inertia was ignored. The length of the hose results in a delay of several seconds before the actuator pressure starts to reduce. It then takes about $400 \mathrm{~s}$ to vanish. The difference between curve 1 and curves 2 and 3 show that distributed effects are clearly important in this situation. However, no significant difference can be seen between 2 and 3 . Hence the premise that inertia of hydraulic oil in the hose is unimportant is shown to be valid, and it can be neglected in this example, because of the very high dissipation number. Because inertia of the oil is negligible, friction will not be frequency dependant.

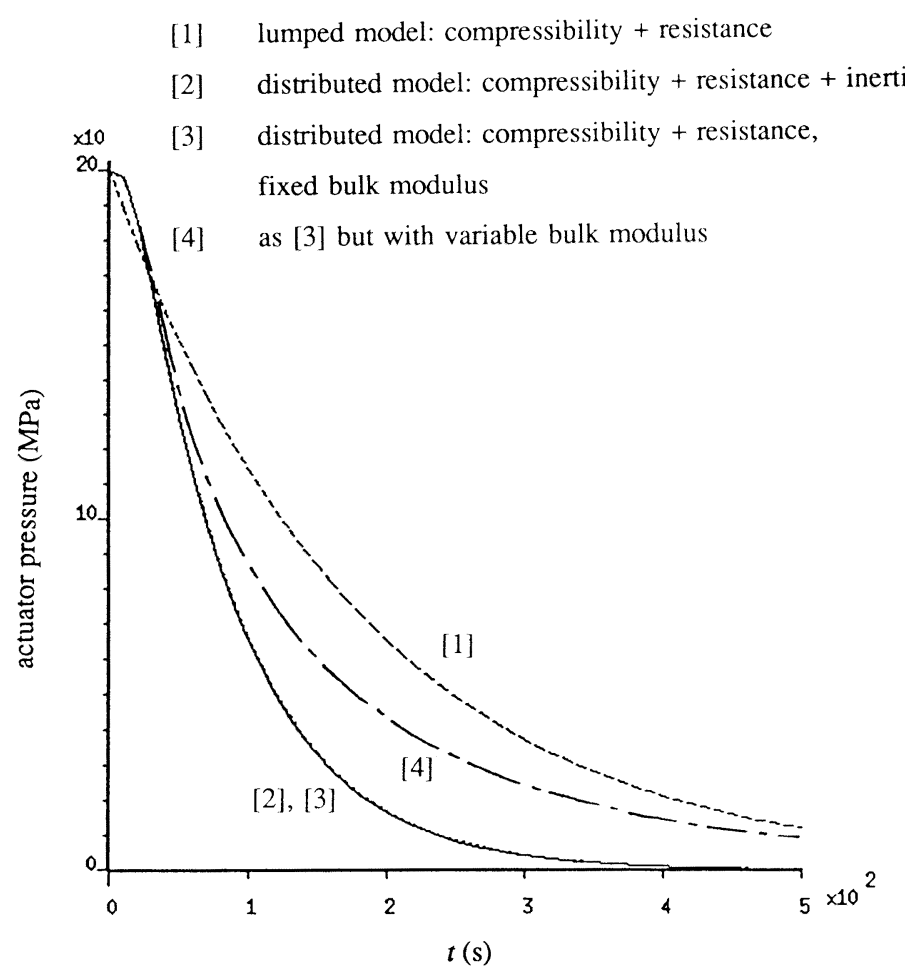

FIGURE 6 Simulated pressure at sea-bed actuator

Simulations were also performed with the effective bulk modulus in the hose dependent upon pressure as described above. Comparing curves 3 and 4 in FIGURE 6 shows that this can have a significant effect. Both curves use the distributed line model ignoring inertia. If the method of characteristics was used in this case, the characteristic lines would vary according to the instantaneous bulk modulus, and this would introduce interpolation error. On the other hand the finite difference method can cope easily with the varying bulk modulus.

\section{Fuel Injection in a Diesel Engine}

A fuel injection system for an internal combustion engine is considered as an example of a system which must operate with extremely rapid transients. A typical arrangement is shown in FIGURE 7 [7,8]. To emphasize the effects of the fuel line, the system is simplified and a single fuel injector is considered. The injector is connected to a plunger pump by a fuel line. A cam pushes the plunger to produce high pressure. When the pressure reaches a sufficient value, a needle opens and fuel is injected into the engine. In the simulation, compressibility of fuel in the plunger pump and in the injector was allowed for. The equation of motion of the needle was used. For the nozzle orifice, a square root relationship between pressure and flow was used, with a constant discharge coefficient. The opening area was varied in proportion to needle lift. The maximum opening area was set to be the total hole area when the needle reached the upper limit. To look at the basic behaviour initially, a simple lumped model with a combination of compressibility and resistance was tested. Simulation results are shown in FIGURE 8. Fuel was injected once in a cycle. This is the ideal behaviour of the fuel injection system.

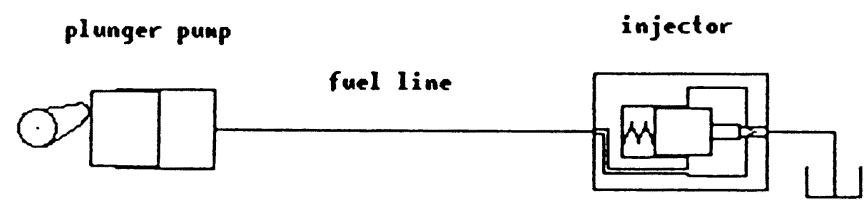

FIGURE 7 Simplified fuel injection system

The fundamental natural frequency of the fuel line is $500 \mathrm{~Hz}$. The plunger lift has a ramp part with a duration of $1.2 \mathrm{~ms}$. From FIGURE 4, the lowest propagation number for the amplitude to become zero is 1.667. The corresponding frequency $(1.667 \times 500)$ is $833 \mathrm{~Hz}$. This propagation number is large enough to excite modes of vibration of the fluid in which the distributed nature of the pipeline is significant, so a distributed model must be used to achieve more accurate simulation. On the other hand, a propagation number of 1.667 would mean that a small number of elements or stations would be adequate. In fact, the first zero in FIGURE 3 still has a fair amount of excitation above it. For greater accuracy one might take the fourth zero, giving an upper frequency limit of $3332 \mathrm{~Hz}$ and a propagation number of 6.67 .

The natural frequency of the spring supported needle is $157 \mathrm{~Hz}$. The upper frequency limit of the fuel line model must cover this frequency as well, but here the speed of the plunger is by far the more stringent requirement. A finite element model was used in which the lengths of the elements were adjusted to optimise the natural frequencies of the model. The upper frequency limit below which the finite element model reproduced the natural frequencies accurately was 3501 

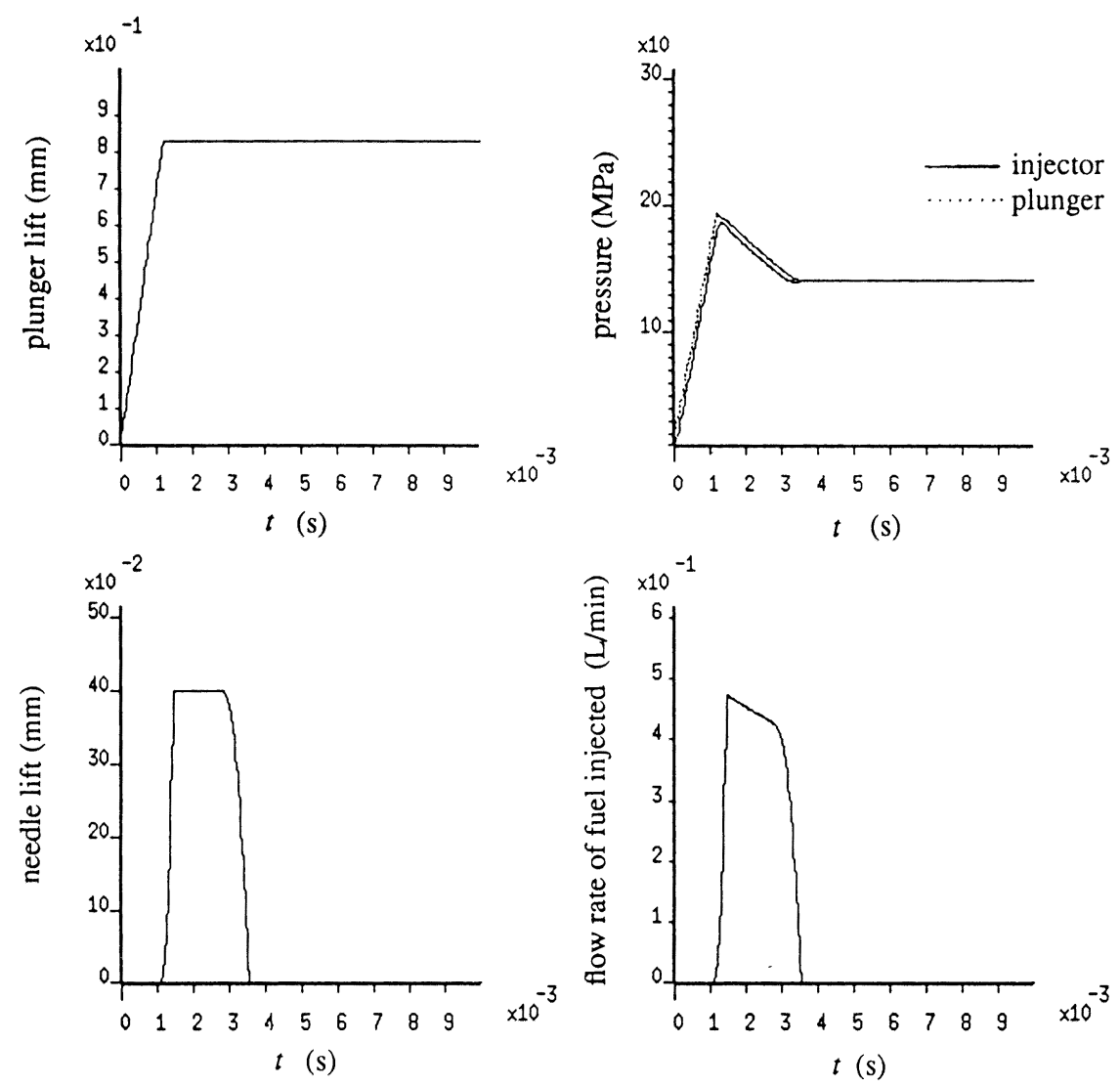

FIGURE 8 Simulated fuel injector characteristics: lumped parameter pipe model
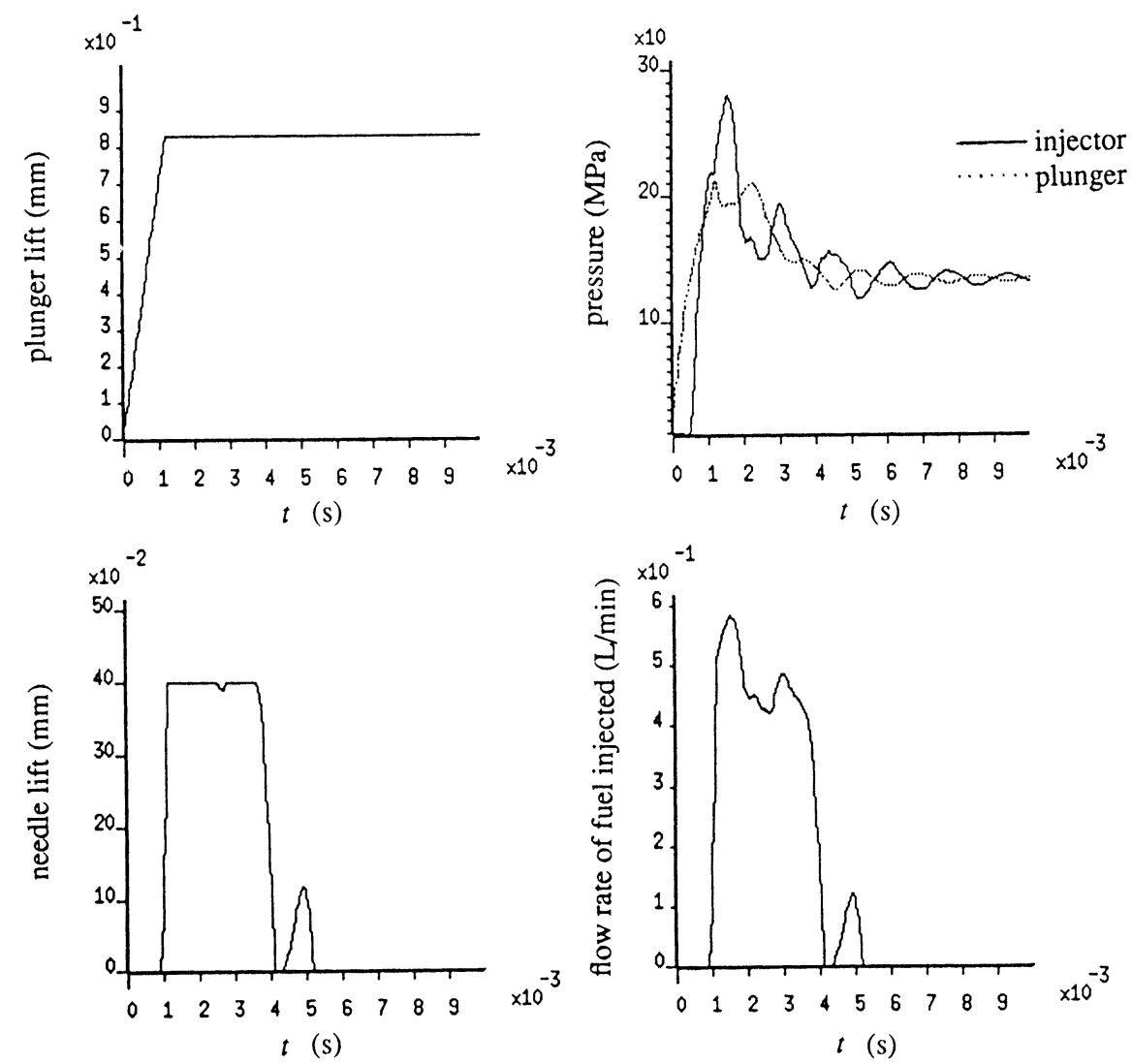

FIGURE 9 Simulated fuel injector characteristics: distributed parameter pipe model 
$\mathrm{Hz}$, which satisfies the above conditions.

The dissipation number of the fuel line is 0.029. From FIGURE 4, the attenuation of waves is significant and cannot be neglected. Frequency dependent friction in laminar flow was modelled.

In addition to the rapid plunger motion, the needle will suddenly stop moving when it reaches the fully open or closed positions. This is another reason for having a model which is accurate up to high frequency. This kind of discontinuity is difficult to simulate accurately. Had the method of characteristics been used, a very small time step would have been needed to catch the discontinuity of the needle movement. Using the optimized finite element model, it is possible to use a well-designed adaptive time step scheme which will accurately capture the instant at which the needle reaches its end stops.

Simulation results by the optimized finite element model with frequency dependent friction are shown in FIGURE 9. As seen from the transient pressures, a pressure wave propagates along the fuel line. Due to the wave propagation, the needle opens twice and the fuel is injected twice. The line pressure increases to a much higher level than was indicated by the simulation using a single lumped model.

\section{CONCLUSIONS}

There are a variety of possible numerical models for simulating pipelines, ranging from ones which involve a single lumped inertia, resistance or compressibility, or a combination of these, through to finite difference and finite element models and the method of characteristics. The frequency content of inputs compared with the lowest natural frequency of the fluid in the pipeline is a useful basis on which to determine whether a distributed model is necessary, and in the case of a finite difference or finite element model, how many stations or elements it should have.

There are many systems in which the frequency content of exciting mechanisms, such as valve closure, is low compared with the natural frequencies of pipelines. Lumped parameter approximations should then be used to minimise computation, and to avoid high frequency transients which would otherwise occur if, for convenience, instantaneous step changes are input into a simulation to represent what are in reality more progressive changes of level.

Even when the frequency content of inputs is high enough to excite pipeline modes, damping may limit the number of modes that need to be modelled accurately. The frequency dependence of fluid friction is particularly helpful in this respect. The low pass filtering effect of other circuit components may also limit the upper frequency range needed for a pipeline model. In very long hoses such as those used for undersea oil rigs, fundamental natural frequencies are so low that it might be expected that large numbers of modes would be excited. However, it has been found that in these circumstances fluid friction and compressibility dominate, and inertia is unimportant.

The method of characteristics can successfully model sudden changes in a pipeline, but if frequency criteria indicate that a comparatively coarse finite difference or finite element model is acceptable, this has the advantage that it can be used with variable time step integration. It is also then easy to have different and varying properties in different elements. An optimised unequal element length model has been found by the authors to improve accuracy in a finite element analysis.

\section{REFERENCES}

1. Wylie, E. B. and Streeter, V. L., Fluid Transients, 1978 (McGraw-Hill).

2. Zielke, W., Frequency-Dependent Friction in Transient Pipe Flow, Trans. ASME, J. Basic Engng., 90, (1), 1968, pp109-115.

3. Yang, W. C. and Tobler, W. E., Dissipative Modal Approximation of Fluid Transmission Lines Using Linear Friction Model, Trans. ASME, J. Dyn. Syst. Measmnt. Contr., 113, March 1991, pp152-162.

4. Watton, J., Fluid Power Systems, Modeling, simulation, analog and microcomputer control, Prentice-Hall, 1989, pp262-265.

5. Paygude, D. G., Vasudeva Rao, B., and Joshi, S. G., Fluid Transients Following a Valve Closure by FEM, Proc. Int. Conf. Finite Elements in Computational Mechanics, Bombay, India, 2-6 Dec. 1985, Pergamon Press, pp615-624.

6. Goodson, R. E. and Leonard, R. G., A Survey of Modeling Techniques of Fluid Line Transients, Trans. ASME, J. Basic Engng, 94(2), 1972, pp474-482.

7. Wassenaar, H., Injection Phenomena in High-speed Diesel Engines, Proc. Auto. Div. Instn Mech Engrs, 1954-1955, pp247-266.

8. Ngugi, S. K., Computer Modelling of Diesel Engine Jerk Pump Fuel Injection Systems, MSc Thesis, University of Bath, UK, 1985 